\title{
Indications of a Si-rich bilateral jet of ejecta in the Vela SNR observed with XMM-Newton
}

\author{
F. García ${ }^{\star, 1,2}$, A. E. Suárez ${ }^{\star}, 1,2$, M. Miceli ${ }^{3,4}$, F. Bocchino ${ }^{4}$, J. A. Combi ${ }^{1,2}$, S. Orlando ${ }^{4}$, and M. Sasaki ${ }^{5}$ \\ 1 Instituto Argentino de Radioastronomía (CCT-La Plata, CONICET; CICPBA), C.C. No. 5, 1894, Villa Elisa, Argentina \\ 2 Facultad de Ciencias Astronómicas y Geofísicas, Universidad Nacional de La Plata, Paseo del Bosque s/n, B1900FWA La Plata, \\ Argentina \\ e-mail: fgarcia@iar-conicet.gov.ar, fgarcia@fcaglp.unlp.edu.ar \\ 3 Dipartimento di Fisica e Chimica, Università degli Studi di Palermo, Piazza del Parlamento 1, 90134 Palermo, Italy \\ 4 INAF-Osservatorio Astronomico di Palermo, Piazza del Parlamento 1, 90134 Palermo, Italy \\ 5 Dr. Karl Remeis-Sternwarte, Erlangen Centre for Astroparticle Physics, Friedrich-Alexander-Universität Erlangen-Nürnberg, \\ Sternwartstrasse 7, 96049 Bamberg, Germany
}

Received 21 June 2017 / Accepted 8 July 2017

\begin{abstract}
Context. The Vela supernova remnant displays several ejecta, which are fragment-like features protruding beyond the front of its primary blast shock wave. They appear to be "shrapnel", bowshock-shaped relics of the supernova explosion. One of these pieces of shrapnel (A), located in the northeastern edge of the remnant, is peculiar because its X-ray spectrum exhibits a high Si abundance, in contrast to the other observed ejecta fragments, which show enhanced $\mathrm{O}, \mathrm{Ne}$, and $\mathrm{Mg}$ abundances.

Aims. In this Letter we present the analysis of another ejecta fragment located opposite to shrapnel A with respect to the center of the shell, in the southwestern boundary of the remnant, named shrapnel G. We aim to fully characterize its X-ray emission to gather new information about the core-collapse supernova explosion mechanism.

Methods. We thoroughly analyzed a dedicated XMM-Newton observation of shrapnel $\mathrm{G}$ by producing background-subtracted and exposure-corrected maps in different energy ranges, which we complemented with a spatially resolved spectral analysis of the X-ray emission.

Results. The fragment presents a bowshock-like shape with its anti-apex pointing to the center of the remnant. Its X-ray spectrum is best fit by a thermal plasma out of equilibrium of ionization with low $\mathrm{O}$ and $\mathrm{Fe}$, roughly solar $\mathrm{Ne}$ and $\mathrm{Mg}$, and a significantly high $\mathrm{Si}$ abundance, which is required to fit a very clear $\mathrm{Si}$ line at $\sim 1.85 \mathrm{keV}$. Its chemical composition and spectral properties are compatible with those of shrapnel A, which is located on the opposite side of the remnant.

Conclusions. As a consequence of the nucleosynthesis, pieces of Si-rich shrapnel are expected to originate in deeper layers of the progenitor star compared to ejecta with lower- $Z$ elements. A high velocity and density contrast with respect to the surrounding ejecta are necessary to make shrapnel A and G overtake the forward shock. The line connecting shrapnel A and G crosses almost exactly the expansion center of the remnant, strongly suggesting a Si-rich jet-counterjet structure, reminiscent of that observed in the young remnant Cas A.
\end{abstract}

Key words. ISM: individual objects: Vela SNR - ISM: supernova remnants - X-rays: ISM

\section{Introduction}

The explosion of a supernova triggered by the collapse of a massive star produces several solar masses of stellar ejecta expanding at $\sim 10^{4} \mathrm{~km} \mathrm{~s}^{-1}$ into the surrounding circumstellar (CSM) and interstellar (ISM) material. The resulting forward shock compresses and heats the gas to high temperatures, thus producing $\mathrm{X}$-ray radiation. As the shock sweeps up material, the deceleration drives a reverse shock back into cold metal-enhanced ejecta, which are also heated to X-ray emitting temperatures. While in young historical supernova remnants (SNRs) the reverse shock is very close to the main blast wave and a significant fraction of the ejecta are still cold and unshocked, the reverse shock in evolved SNRs has had time to reach the SNR center, and therefore all the ejecta has been shocked and is expected to emit X-rays.

Several SNRs are characterized by a knotty ejecta structure, and very many clumps have been observed at different

\footnotetext{
^ Fellow of CONICET, Argentina.
}

wavelengths in remnants of core-collapse supernovae ( $\mathrm{SNe}$ ), such as G292.0+1.8 (Park et al. 2004), Puppis A (Katsuda et al. 2008a), and Cas A, where knots have also been detected beyond the main shock front (Hammell \& Fesen 2008; DeLaney et al. 2010).

The Vela SNR represents a privileged target for studying the distribution of the ejecta and fragments detected beyond the forward shock front. It is considered to be the remnant of a Type II-P SN explosion of a progenitor star with a mass lower than $25 M_{\odot}$ (Gvaramadze 1999). Its age is estimated to be $11.4 \mathrm{kyr}$ (Taylor et al. 1993), and the distance to the SNR is about 250 pc (Bocchino et al. 1999; Cha et al. 1999). Aschenbach et al. (1995) identified six "shrapnel" (labeled shrapnel A-F), which are X-ray emitting ejecta fragments with a characteristic boomerang shape protruding beyond the primary blast wave. Shrapnel A, B, and D have been studied in detail by Tsunemi et al. (1999), Miyata et al. (2001), Katsuda \& Tsunemi (2005, 2006) and Yamaguchi \& Katsuda (2009). These works 


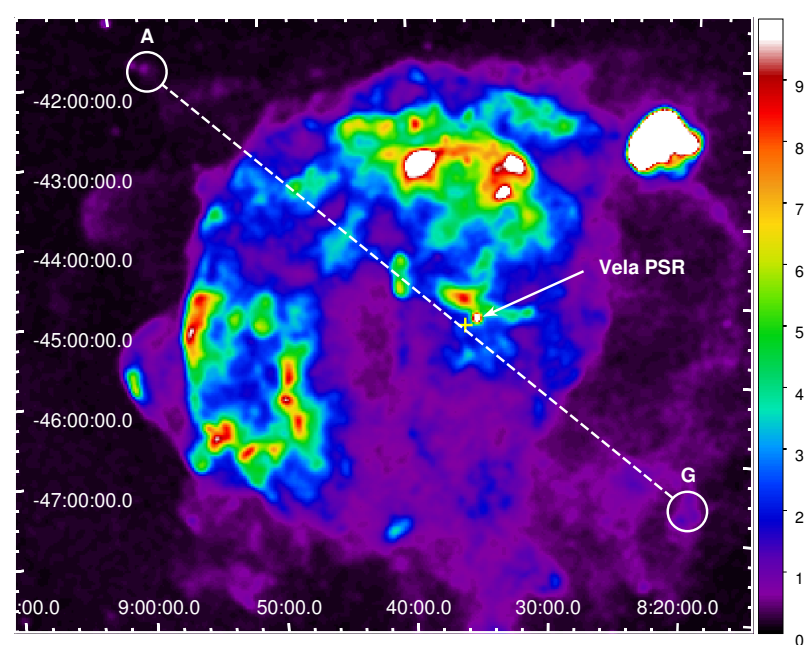

Fig. 1. ROSAT All-Sky Survey image of the Vela SNR in the 0.44-2.04 keV energy range. Shrapnel A and G are indicated with white circles connected by a dashed line that crosses close to the Vela PSR, and the explosion point inferred from its age and proper motion (yellow cross).

have shown that the shrapnel may be divided into two categories. Shrapnel B and D have high $\mathrm{O}, \mathrm{Ne}$, and $\mathrm{Mg}$ abundances, while shrapnel A has a high Si abundance and weak emission from other elements. Consequently, Tsunemi \& Katsuda (2006) have pointed out that the Si-rich shrapnel A must have been generated in a deeper layer of the progenitor than all the other shrapnel. Several bright ejecta knots have been discovered in the northern part of the remnant (Miceli et al. 2008). The authors suggested that these knots would be shrapnel hidden inside the main shell by a projection effect, showing relative abundances similar to those found in shrapnel B and D.

In other core-collapse SNRs, the Si-rich ejecta may show a very peculiar jet-counterjet structure. Moreover, Grichener \& Soker (2017) suggested that jet-like features are common in many core-collapse SNRs. The well-known case of Cas A has been studied in detail thanks to a very long Chandra observation (Hwang et al. 2004) that shows a jet (with a weaker counterjet structure) composed mainly of Si-rich plasma. Laming et al. (2006) have performed an X-ray spectral analysis of several knots in the jet and concluded that the origin of this interesting morphology lies in an explosive jet and it does not arise through interaction with a cavity or other peculiar structure of the ISM or CSM. Detailed 3D hydrodynamic simulations have shown that this jet can be explained as the result of velocity and density inhomogeneities in the ejecta profile of the exploding star with $\sim 2 \%$ of the energy of the total energy budget of this remnant (Orlando et al. 2016).

In this Letter we present the analysis of an XMM-Newton dedicated observation of shrapnel $\mathrm{G}$, located in the southwestern edge of the Vela SNR. We investigate the Si-rich ejecta in this shrapnel, which lies on the same line as the line that connects the center of the shell and the northeastern shrapnel A in the plane of the sky (see Fig. 1). Shrapnel A and G reveal the signature of a jet-counterjet $\mathrm{Si}$-rich structure in the ejecta of the very old Vela SNR, reminiscent of the very young core-collapse SNR Cas A.

\section{Observations and data analysis}

The Vela shrapnel $\mathrm{G}$ has been observed once with the European Photon Imaging Camera (EPIC) of the XMM-Newton satellite.
This camera consists of three detectors, two MOS cameras, namely MOS1 and MOS2 (Turner et al. 2001), and a PN camera (Strüder et al. 2001), which operate in the $0.3-10 \mathrm{keV}$ energy range. The observation was performed on April 22, 2012 (Obs. ID 0675080101), with a medium filter in Prime Full Window observation mode. The exposures were $42 \mathrm{ks}, 43 \mathrm{ks}$, and $41 \mathrm{ks}$ for the MOS1, MOS2, and PN cameras, respectively.

We analyzed the data using XMM-Newton Science Analysis System (SAS) version 15.0.0 and calibration files available in December 2016. In order to avoid soft-proton contamination, the observations were filtered using the SAS task ESPFILT, resulting in reduced Good Time Intervals (GTI) of approximately $28 \mathrm{ks}$ for MOS1, $29 \mathrm{ks}$ for MOS2, and $27 \mathrm{ks}$ for PN exposures. For the subsequent analysis, the event lists were filtered to retain only events that likely stem from X-ray photons: we selected FLAG $==0$ events with single and double PATTERN by means of the EVSELECT task.

\section{Results}

\subsection{X-ray images}

To produce images in different energy bands, we performed a double background subtraction to take into account particle and X-ray background contamination. For this purpose, we used Filter Wheel Closed and Blank Sky files available at XMM ESAC webpages ${ }^{1}$ and adopted the procedure described in Miceli et al. (2017). We then performed a point-source detection by running the EDETECT_CHAIN script. Events in circular regions of 15 arcsec around each detected source were removed from the filtered event files, as we are only interested in the diffuse emission of the shrapnel. We created background-subtracted images for each camera correcting for exposure and vignetting effects in different energy bands. Finally, we combined them by applying an adaptive smoothing by means of the EMOSAIC and ASMOOTH tasks.

In Fig. 2 we show the resulting composite X-ray image of the Vela shrapnel $G$ obtained by combining the three EPIC exposures using a spatial binning of 4 arcsec. The soft band $(0.3-0.6 \mathrm{keV})$ is shown in red, the medium band $(0.6-1.3 \mathrm{keV})$ in green, and the hard (1.3-3.0 keV) band in blue. Black circles correspond to the subtracted point-sources. In the image, north is up and east is to the left. The shrapnel is indistinguishable from the background above $3.0 \mathrm{keV}$ in the available data. As can be seen, the morphology of the shrapnel is fairly regular, showing two bright extended eastern (E) and western (W) regions. The $\mathrm{X}$-ray emission shows a strong edge to the southwest (SW) coincident with a possible shock front of the shrapnel and weak elongated X-ray emission pointing to the northeast (NE), corresponding to the geometrical center of the Vela SNR.

In Fig. 3 we show a mosaiced X-ray map of the Si band $(1.3-2.0 \mathrm{keV})$ with a spatial binning of 20 arcsec. The overlaid yellow contours correspond to the X-ray emission in the whole $0.3-3.0 \mathrm{keV}$ energy range. From this map it is evident that the photons originated in the Si band are spatially correlated with the total X-ray emission.

\footnotetext{
1 https://wwW. cosmos. esa.int/web/xmm-newton/ filter-closed,

http://xmm-tools. cosmos. esa.int/external/xmm calibration/\background/bs_repository/blanksky_all. html
} 


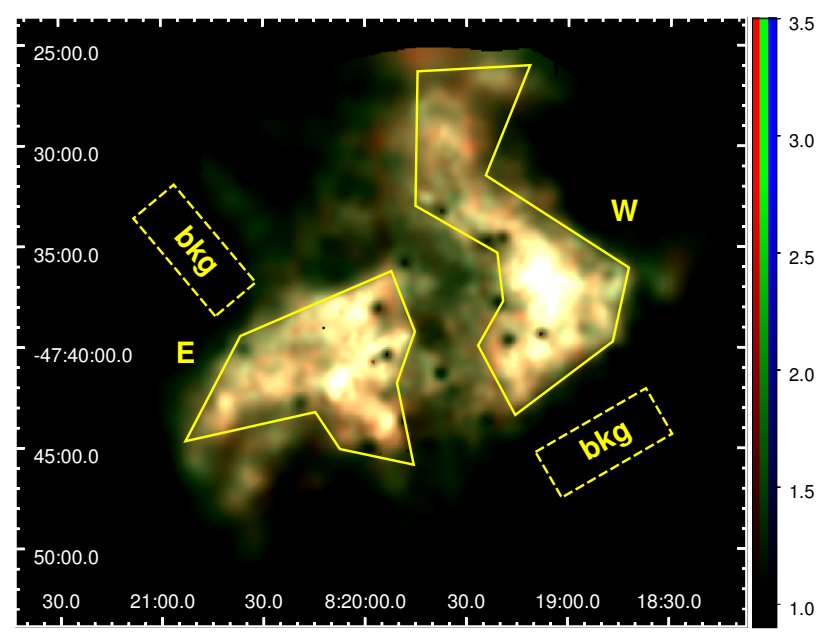

Fig. 2. XMM-Newton false-color count-rate image of Vela shrapnel G. Red represents $0.3-0.6 \mathrm{keV}$, green $0.6-1.3 \mathrm{keV}$, and blue the $1.3-3.0 \mathrm{keV}$ energy range. The overlaid yellow contours indicate the east (E) and west (W) spectral extraction regions. Dashed rectangles indicate the background regions (bkg). The image is background- and vignetting-corrected and point sources were removed.

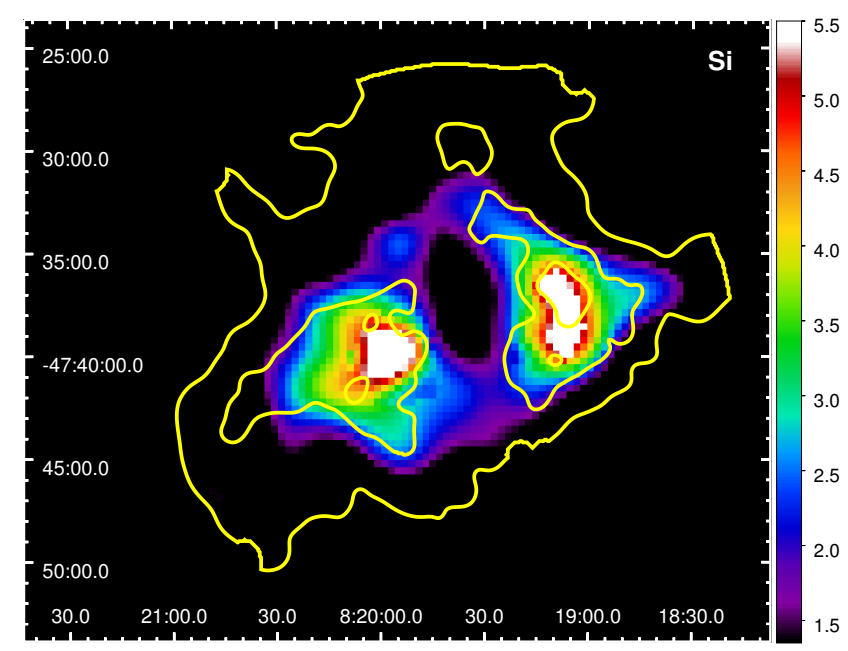

Fig. 3. Background- and vignetting-corrected count-rate map in the 1.3-2.0 keV energy range, corresponding to the Si band. The overlaid yellow contours represent the emission in the $0.3-3.0 \mathrm{keV}$ range. The Si maxima match the two bright knots of X-ray emission well.

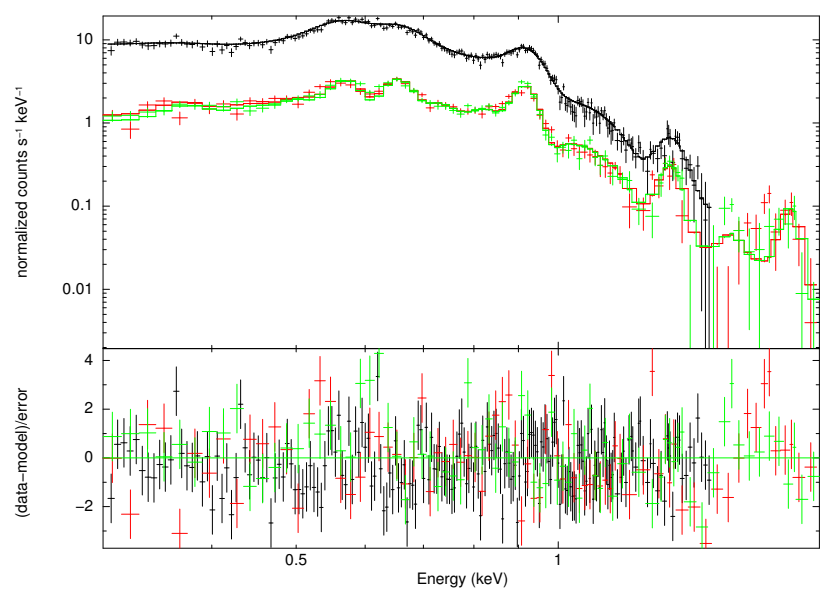

Fig. 4. PN and MOS1/2 spectra of shrapnel G. Solid lines indicate the best-fit VNEI model (see Table 1). Lower panel shows the fit residuals.
Table 1. Spectral parameters of Vela shrapnel G.

\begin{tabular}{l|c|c}
\hline \hline Model \& Parameters & VAPEC+VAPEC & VNEI \\
\hline$N_{\mathrm{H}}\left[10^{22} \mathrm{~cm}^{-2}\right]$ & $0.11 \pm 0.01$ & $0.022 \pm 0.007$ \\
\hline$k T_{1}[\mathrm{keV}]$ & $0.194 \pm 0.002$ & $0.49 \pm 0.02$ \\
$\mathrm{Norm}_{1}\left[\times 10^{-3}\right]$ & $25 \pm 3$ & $2.0 \pm 0.3$ \\
$\tau\left[10^{10} \mathrm{~s} \mathrm{~cm}^{-3}\right]$ & - & $3.1 \pm 0.3$ \\
\hline$k T_{2}[\mathrm{keV}]$ & $0.64 \pm 0.07$ & - \\
$\mathrm{Norm}_{2}\left[\times 10^{-3}\right]$ & $0.66 \pm 0.08$ & - \\
\hline $\mathrm{O},(=\mathrm{N}),(=\mathrm{C})\left[\mathrm{O}_{\odot}\right]$ & $0.38 \pm 0.02$ & $0.47 \pm 0.05$ \\
$\mathrm{Ne}\left[\mathrm{Ne}_{\odot}\right]$ & $1.14 \pm 0.08$ & $1.33 \pm 0.10$ \\
$\mathrm{Mg}\left[\mathrm{Mg}_{\odot}\right]$ & $0.99 \pm 0.11$ & $0.92 \pm 0.12$ \\
$\mathrm{Si}\left[\mathrm{Si}_{\odot}\right]$ & $2.06 \pm 0.45$ & $2.24 \pm 0.43$ \\
Fe $\left[\mathrm{Fe}_{\odot}\right]$ & $0.34 \pm 0.03$ & $0.29 \pm 0.04$ \\
\hline$\chi_{v}^{2} / \mathrm{d}_{0} . \mathrm{o.f}$. & $1.50 / 450$ & $1.41 / 451$ \\
\hline Flux $(0.3-0.6 \mathrm{keV})$ & $4.49 \pm 0.06$ & $4.57 \pm 0.06$ \\
Flux $(0.6-1.3 \mathrm{keV})$ & $4.75 \pm 0.03$ & $4.73 \pm 0.03$ \\
Flux $(1.3-3.0 \mathrm{keV})$ & $0.33 \pm 0.02$ & $0.29 \pm 0.01$ \\
\hline Total Flux $(0.3-3.0 \mathrm{keV})$ & $9.57 \pm 0.06$ & $9.59 \pm 0.06$ \\
\hline
\end{tabular}

Notes. Normalizations are defined as $10^{-14} / 4 \pi \mathrm{D}^{2} \times E M$, where $E M=$ $\int n_{\mathrm{H}} n_{\mathrm{e}} \mathrm{d} V$ is the emission measure, $D$ is distance in [cm], $n_{\mathrm{H}}$ and $n_{\mathrm{e}}$ are the hydrogen and electron densities $\left[\mathrm{cm}^{-3}\right]$, and $V$ is the volume $\left[\mathrm{cm}^{3}\right]$. Considering $D=250 \mathrm{pc}$ and a solid angle $\Omega=A / D^{2}=1.17 \times 10^{-5} \mathrm{sr}$ for the spectral regions, we obtain an $E M / A=2.1 \times 10^{17} \mathrm{~cm}^{-5}$ for the preferred VNEI model. Error values are $1 \sigma(68 \%)$ confidence intervals for each free parameter. Fluxes are given in units of $10^{-12} \mathrm{erg} \mathrm{cm}^{-2} \mathrm{~s}^{-1}$ and solar abundances are taken from Anders \& Grevesse (1989).

\section{2. $X$-ray spectra}

Since the diffuse emission from the shrapnel G, which is an inhomogeneous extended source, occupies almost the full field of view, we applied the SAS EVIGWEIGHT task to correct the event lists for vignetting effects. We obtained response and ancillary matrices using RMFGEN and ARFGEN for a flat detector map, and we binned the spectra to obtain at least 25 counts per bin. We selected two polygonal spectral extraction regions named $\mathrm{E}$ and $\mathrm{W}$, and background was extracted from two different regions where no significant emission from the shrapnel was detected (see Fig. 2). The spectral analysis was performed using the XSPEC package (version 12.9.0, Arnaud 1996) in the 0.3-2 keV band for the two MOS cameras and in the $0.3-1.5 \mathrm{keV}$ band for the pn. The pn spectrum above $1.5 \mathrm{keV}$ is dominated by the background, so we did not include it in our analysis to maximize the signal-to-noise ratio. We verified that our best-fit model does not change significantly by adding the pn data in the $1.5-2 \mathrm{keV}$ band, although the indetermination of the best-fit parameters increases. Since we did not find a significant difference between the spectral fits of regions $\mathrm{E}$ and $\mathrm{W}$, we combined them to improve the signal-to-noise ratio. Furthermore, we also checked that independently of the background region used, the best-fit values were consistent with each other. We inspected the background spectra and verified that they do not show any feature at the $1.85 \mathrm{keV}$ Si band, which is visible only in the source spectra.

In Fig. 4 we show the background-subtracted X-ray spectra of the Vela shrapnel G for the three EPIC cameras (black for PN, red and green for MOS 1 and MOS 2, respectively). Errors are at $1 \sigma(68 \%)$ confidence levels, and $\chi^{2}$ statistics are used. The X-ray spectrum of shrapnel $G$ has a thermal origin, showing emission lines from O VII $(0.56 \mathrm{keV})$, 
O VIII (0.65 keV), Ne IX (0.92 keV), Mg XI (1.35 keV), and Si $(1.85 \mathrm{keV})$. We fit the spectra with different models of thermal emission from an optically thin plasma in collisional ionization equilibrium (APEC model) and in non-equilibrium of ionization (NEI model), taking into account the interstellar absorption (PHABS, Balucinska-Church \& McCammon 1992). The best fit is obtained for an NEI plasma with $k T=0.49 \pm 0.02 \mathrm{keV}$. A two-temperature APEC plasma with $k T_{1}=0.194 \pm 0.02 \mathrm{keV}$ and $k T_{2}=0.64 \pm 0.07 \mathrm{keV}$ also gives a good fit. Non-solar abundances are required for both models to fit the emission lines, and their values are compatible between each other. The bestfit parameters are presented in Table 1. From now on, we focus on the single-temperature NEI model, which provides a significantly better fit to the spectra.

Before this study, a bright Si He $\alpha$ emission line could be detected only in the northeastern shrapnel A (Katsuda \& Tsunemi 2006). Remarkably, shrapnel $G$ is located in the opposite southwestern region of shrapnel A. In contrast, shrapnel D (Katsuda \& Tsunemi 2005) and the ejecta knots found by Miceli et al. (2008) in the northern rim of Vela SNR showed no $\mathrm{Si}$ line and notably higher abundances of $\mathrm{O}, \mathrm{Ne}, \mathrm{Mg}$ and $\mathrm{Fe}$ than those found in shrapnel A (Katsuda \& Tsunemi 2006) and $G$ (this study). In particular, we found that in shrapnel $G$ the $\mathrm{Ne}: \mathrm{Mg}: \mathrm{Si}: \mathrm{Fe}: \mathrm{O}$ abundances relative to $\mathrm{O}$ are 2.8:2.0:4.8:0.6:1 to be compared with 2.6:2.2:7.6:2.6:1 in shrapnel A, while shrapnel $\mathrm{D}$ and northern ejecta present $\mathrm{Ne}: \mathrm{Mg}: \mathrm{Fe}: \mathrm{O}=2.1: 2.2: 0.2: 1$ and 2.5:3.2:0.5:1 and no Si line (Miceli et al. 2008). Similar abundance patterns have also been observed by LaMassa et al. (2008), who found ejecta-rich plasma in the (projected) direction of the Vela PSR. In conclusion, shrapnel A and G are the only Si-rich shrapnel observed up to now in the Vela SNR. Furthermore, their plasma temperatures are consistent within $2 \sigma$, and the ionization parameters are quite similar (see Table 1 in Katsuda \& Tsunemi 2006), as are their projected sizes in the plane of the sky.

\section{Discussion}

In this Letter we presented a detailed study of the shrapnel G located in the SW region of the Vela SNR and showed the presence of Si-rich plasma in shrapnel $\mathrm{G}$ for the first time. We showed that shrapnel G has a very similar chemical composition to the geometrically opposite shrapnel A located in the NE edge of the remnant. While shrapnel B and D and all the other ejecta knots detected so far in Vela SNR have high $\mathrm{O}, \mathrm{Ne}$, and $\mathrm{Mg}$ abundances, shrapnel A and G have a high Si abundance and weak emission from other elements.

As a consequence of the nucleosynthesis expected in stellar evolution, Tsunemi \& Katsuda (2006) pointed out that Si-rich shrapnel must be generated in deeper layers of the progenitor star. However, hydrodynamic simulations of the Vela shrapnel show that an unrealistically high initial density contrast is required for an inner shrapnel to overcome outer ejecta knots, if we assume that the ejecta velocity increases linearly with their distance from the center (Miceli et al. 2008). A possible solution for this issue is that (part of) the Si-burning layer has been ejected with a higher initial velocity, for example, as a collimated jet. This idea was later confirmed by dedicated 3D simulations of Cas A, showing that both density and velocity inhomogeneities are necessary to reproduce the observed Si-rich jet (Orlando et al. 2016).

The line connecting shrapnel A and G passes almost exactly through the expansion center of Vela SNR (see Fig. 1) as determined from the geometry of the other shrapnel and the proper motion of the Vela PSR. This alignment strongly supports the possibility that these shrapnel pieces are part of a Si-rich jetcounterjet structure. Assuming that the size along the line of sight, $L$, is equal to the projected size of shrapnel $\mathrm{G}$ in the plane of the sky $(1320 \mathrm{arcsec})$ at a distance of $D=250 \mathrm{pc}$, we estimate a number density of $n=0.21 \mathrm{~cm}^{-3}$ for the X-ray emitting plasma, and a total mass of $M=0.008 M_{\odot}$ (for an average atomic mass of $2.1 \times 10^{-24} \mathrm{~g}$ for solar abundances). Considering the projected distance from shrapnel $G$ to the geometrical center of the SNR and an age of $\sim 11 \mathrm{kyr}$, we obtain a velocity of $\sim 1400 \mathrm{~km} \mathrm{~s}^{-1}$, leading to a total average kinetic energy of $E=1.6 \times 10^{47} \mathrm{erg}$, which is a lower limit when we take into account that the velocity of the shrapnel is not constant in time, that our estimate accounts only for the projected velocity, and that the X-ray emitting mass is only a fraction of the initial mass (Miceli et al. 2013). Interestingly, the estimated mass of shrapnel $\mathrm{G}$ is similar (within a factor of 5) to the mass of the postexplosion anisotropy responsible for the $\mathrm{Si}$-rich jet observed in Cas A (Orlando et al. 2016). On the other hand, our lower limit on the kinetic energy is two orders of magnitude lower than the energy estimated soon after the SN explosion for the jet of Cas A $\left(\sim 4 \times 10^{49} \mathrm{erg}\right.$, Orlando et al. 2016). This is mainly due to our estimated velocity of shrapnel G: a velocity higher by a factor of 10 (which is expected soon after the SN explosion) would produce energies similar to the energy found in the jet of Cas A. We conclude that the mass and energy inferred for shrapnel $G$ is very similar to the values estimated for the jet of Cas A (Orlando et al. 2016).

The structure of the ejecta in a SNR contains the imprint of the metal-rich layers inside the progenitor star. This type of detailed analysis of spatially resolved ejecta may help to understand the processes occurring in the latest stage of stellar evolution on the onset of core-collapse SNe explosions. In this sense, Vela SNR is an ideal candidate for performing this type of studies because of its age and angular size in the sky. Dedicated observations in the X-ray band of the remaining shrapnel that has not been studied so far are required to probe the still poorly understood physics of core-collapse supernovae and the formation of collimated ejecta jets.

With existing X-ray telescopes like XMM-Newton, it is possible to study small parts of a large SNR like Vela in pointed observations. Since we are not able to cover the entire SNR, it is difficult to achieve an understanding of the object as a whole. The German telescope eROSITA (extended ROentgen Survey with an Imaging Telescope Array, Merloni et al. 2012) on board the Russian Spektrum-Roentgen-Gamma (SRG) mission, which is planned to be launched in 2018, will perform an all-sky survey (eRASS) in the $0.3-10 \mathrm{keV}$ band for the first time. Equipped with CCDs similar to those of XMM-Newton, we will be able to study the entire SNR with a similar spatial and spectral resolution as we have presented here for shrapnel G. With a total exposure of $\sim 3 \mathrm{ks}$, eRASS will yield $\sim 23000$ net counts for shrapnel $\mathrm{G}$, allowing us to constrain abundances with an accuracy of $\sim 20 \%$.

Acknowledgements. We are grateful to the referee for very constructive comments. The research leading to these results has received funding from the European Union Horizon 2020 Programme under the AHEAD project (grant agreement n. 654215). FG and AES are grateful for the hospitality of INAF Osservatorio Astronomico di Palermo members. F.G., A.E.S. and J.A.C. were supported by PIP 0102 (CONICET). JAC was also supported by Consejería de Economía, Innovación, Ciencia y Empleo of Junta de Andalucía under grant FQM-1343, and research group FQM-322, as well as FEDER funds. M.M. and S.O. aknowledge support by the PRIN INAF 2014 grant "Filling the gap between supernova explosions and their remnants through magnetohydrodynamic 
F. García et al.: Indications of a Si-rich bilateral jet of ejecta in the Vela SNR observed with XMM-Newton

modeling and high performance computing". MS acknowledges support by the Deutsche Forschungsgemeinschaft (DFG) through the Heisenberg professor grant SA 2131/5-1.

\section{References}

Anders, E., \& Grevesse, N. 1989, Geochim. Cosmochim. Acta, 53, 197 Arnaud, K. A. 1996, ASP Conf. Ser., 101, 17

Aschenbach, B., Egger, R., \& Trümper, J. 1995, Nature, 373, 587 Balucinska-Church, M., \& McCammon, D. 1992, ApJ, 400, 699 Bocchino, F., Maggio, A., \& Sciortino, S. 1999, A\&A, 342, 839 Cha, A. N., Sembach, K. R., \& Danks, A. C. 1999, ApJ, 515, L25 DeLaney, T., Rudnick, L., Stage, M. D., et al. 2010, ApJ, 725, 2038

Hammell, M. C., \& Fesen, R. A. 2008, ApJS, 179, 195

Grichener, A., \& Soker, N. 2017, MNRAS, 468, 1226

Gvaramadze, V. 1999, A\&A, 352, 712

Hwang, U., Laming, J. M., Badenes, C., et al. 2004, ApJ, 615, L117
Katsuda, S., Mori, K., Tsunemi, H., et al. 2008, ApJ, 678, 297 Katsuda, S., \& Tsunemi, H. 2005, PASJ, 57, 621 Katsuda, S., \& Tsunemi, H. 2006, ApJ, 642, 917 LaMassa, S. M., Slane, P. O., \& de Jager, O. C. 2008, ApJ, 689, L121 Laming, J. M., Hwang, U., Radics, B., et al. 2006, ApJ, 644, 260 Merloni, A., Predehl, P., Becker, W., et al. 2012, ArXiv e-prints [arXiv: 1209.3114]

Miceli, M., Bocchino, F., \& Reale, F. 2008, ApJ, 676, 1064

Miceli, M., Orlando, S., Reale, F., et al. 2013, MNRAS, 430, 2864

Miceli, M., Bamba, A., Orlando, S., et al. 2017, A\&A, 599, A45

Miyata, E., Tsunemi, H., Aschenbach, B., \& Mori, K. 2001, ApJ, 559, L45 Orlando, S., Miceli, M., Pumo, M. L., \& Bocchino, F. 2016, ApJ, 822, 22 Park, S., Hughes, J. P., Slane, P. O., et al. 2004, ApJ, 602, L33

Strüder, L., Briel, U., Dennerl, K., et al. 2001, A\&A, 365, L18 Taylor, J. H., Manchester, R. N., \& Lyne, A. G. 1993, ApJS, 88, 529 Tsunemi, H., Miyata, E., \& Aschenbach, B. 1999, PASJ, 51, 711 Tsunemi, H., \& Katsuda, S. 2006, New Astron., 50, 521 Turner, M. J. L., Abbey, A., Arnaud, M., et al. 2001, A\&A, 365, L27 Yamaguchi, H., \& Katsuda, S. 2009, ApJ, 696, 1548 